\title{
UNIQUENESS OF THE INVARIANT MEAN ON ABELIAN TOPOLOGICAL SEMIGROUPS
}

BY

\author{
INDAR S. LUTHAR
}

Let $S$ be a topological semigroup $\left(^{1}\right)$ and let $C(S)$ be the space of bounded continuous real-valued functions $x$ on $S$ with

$$
\|x\|=\operatorname{Sup}_{\sigma \in S}|x(\sigma)| \text {. }
$$

For each $\sigma \in S$ we define the left translation operator

$$
l_{\sigma}: C(S) \rightarrow C(S)
$$

by

$$
\left(l_{\sigma} x\right)(\tau)=x(\sigma \tau)
$$

and the right translation operator

$$
r_{\sigma}: C(S) \rightarrow C(S)
$$

by

$$
\left(r_{\sigma} x\right)(\tau)=x(\tau \sigma) .
$$

An element $\nu \in C(S)^{*}$ is called a mean if

$$
\|\nu\|=1=\nu(e)
$$

where $e$ is the function which is identically one. An element $\nu$ in $C(S)^{*}$ is called left-invariant if $l_{\sigma}^{*} \nu=\nu$ for every $\sigma$ in $S$ and is called right-invariant if $r_{\sigma}^{*} \nu=\nu$ for every $\sigma \in S$. We say that $\nu$ is invariant if $l_{\sigma}^{*} \nu=\nu=r_{\sigma}^{*} \nu$ for every $\sigma$ in $S$. $S$ is called amenable if there exists an invariant mean. It is known that an Abelian topological semigroup is amenable.

In my earlier paper [2] I proved that a discrete Abelian semigroup has a unique invariant mean if and only if it has a finite ideal. It is quite reasonable to conjecture that in general an Abelian topological semigroup has a unique invariant mean if and only if the semigroup has a compact ideal. In this paper we prove the conjecture in certain special situations.

TheOREM 1(2). An abelian topological semigroup with a compact ideal has a unique invariant mean.

Received by the editors April 11, 1961 and, in revised form, October 30, 1961.

(1) By a topological semigroup we mean a semigroup provided with a Hausdorff topology in which the mapping $(\sigma, \tau) \rightarrow \sigma \tau$ of $S \times S$ into $S$ is continuous.

(2) In the original manuscript this theorem was proved under the assumption that the semigroup is normal. The author is grateful to the referee for suggesting a method for removing the condition of normality. 
Proof. Let $\Delta$ be a compact ideal of $S$. If $\Delta_{1}, \cdots, \Delta_{n}$ be closed ideals of $S$, each contained in $\Delta$, then

$$
\Delta^{*}=\bigcap_{i=1}^{n} \Delta_{i} \supset \Delta_{1} \cdots \Delta_{n}
$$

and so is nonempty. Thus the family $\mathcal{F}$ of all closed ideals of $S$ contained in $\Delta$ has the finite intersection property and so due to the compactness of $\Delta$

$$
A=\bigcap_{\Delta^{\prime} \in \mathcal{F}} \Delta^{\prime} \neq \varnothing \text {. }
$$

Thus $A$ is an ideal. This $A$ is obviously a minimal compact ideal of $S$. Take any $a \in A$. Then $a A$ is an ideal contained in $A$ and so $a A=A$ due to the minimal character of $A$. Therefore $A$ is a group.

We now define a relation among the elements of $A$. We say that two elements $a$ and $a^{\prime}$ in $A$ are equivalent if they cannot be separated by a continuous function on $S$, i.e., if $x(a)=x\left(a^{\prime}\right)$ for every $x$ in $C(S)$. This is obviously an equivalence relation. Let $H$ be the set of elements of $A$ which are equivalent to the identity $e$ of the group $A$. We claim that $H$ is a closed subgroup of $A$ and that the equivalence classes are simply the cosets of $H$ in $A$. That $H$ is closed follows from the fact that

$$
H=\bigcap_{x \in C(S)}\{a \mid a \in A, x(a)=x(e)\} .
$$

To prove the remaining assertion we proceed as follows:

For each $\sigma \in S$, we define $\hat{\sigma}$ by

$$
\hat{\sigma}(s)=\sigma s ; s \in S .
$$

If $a$ and $b$ are equivalent then $a b^{-1}$ and $e$ are equivalent, for

$$
x\left(a b^{-1}\right)=\left(x \circ\left(b^{-1}\right)^{\wedge}\right)(a)=\left(x \circ\left(b^{-1}\right)^{\wedge}\right)(b)=x(e) .
$$

Conversely the equivalence of $a b^{-1}$ and $e$ implies the equivalence of $a$ and $b$, since

$$
x(a)=(x \circ \hat{b})\left(a b^{-1}\right)=(x \circ \hat{b})(e)=x(b) .
$$

From this can be easily deduced the assertions made above.

We now form the quotient group $A / H$. Each $x$ in $C(S)$ defines an $\tilde{x}$ in $C(A / H)$. It is obvious that if $\tilde{x}_{1}=\tilde{x}_{2}$ then $x_{1}$ and $x_{2}$ agree on $A$.

The set $\tilde{C}=\{\tilde{x} \mid x \in C(S)\}$ separates the points of $A / H$. Since $A / H$ is compact the set $\widetilde{C}$ is dense in $C(A / H)$ by the Stone-Weierstrass Theorem.

Let $\nu$ be any invariant mean on $C(S)$. If $z$ is in $C(S)$ we define

$$
\|z\|_{A}=\operatorname{Sup}_{a \in A}|z(a)| \text {. }
$$

If $z_{\lambda}, \lambda \in \Lambda$ is a net of elements of $C(S)$ such that $\left\|z_{\lambda}\right\| A_{A} \rightarrow 0$ then $\nu\left(z_{\lambda}\right) \rightarrow 0$ for $\left|\nu\left(z_{\lambda}\right)\right|=\left|\nu\left(l_{a} z_{\lambda}\right)\right| \leqq\left\|l_{a} z_{\lambda}|| \leqq\right\| z_{\lambda} \|_{A}$ where $a$ is any fixed element of $A$. 
These facts allow us to define invariant integration on $A / H$ as follows:

Let $y \in C(A / H)$. We can choose $\tilde{x}_{n}$ in $\tilde{C}$ such that $\tilde{x}_{n} \rightarrow y$. It is obvious that $\left\|x_{m}-x_{n}\right\|_{A} \rightarrow 0$ as $m, n \rightarrow \infty$ and so $\nu\left(x_{m}-x_{n}\right) \rightarrow 0$ as $m, n \rightarrow \infty$. Thus $\nu\left(x_{n}\right)$ is a Cauchy sequence and so possesses a unique limit. This limit depends only on $y$ since for any other sequence $\tilde{z}_{n}$ converging to $y$ we have

$$
\left\|\tilde{x}_{n}-\tilde{z}_{n}\right\| \rightarrow 0 .
$$

Thus $\left\|x_{n}-z_{n}\right\|_{A} \rightarrow 0$ and so $\lim \nu\left(x_{n}\right)=\lim \nu\left(z_{n}\right)$. We define

$$
\int_{A / H} y=\lim _{n} \nu\left(x_{n}\right) .
$$

That the integral is invariant follows from the fact that for any $\bar{a}$ in $A / H$, $\bar{l}_{\tilde{a}}(\tilde{x})=\left(l_{a} x\right)^{\sim}$ and that $\nu\left(l_{a} x\right)=\nu(x)$. Since $A / H$ is a compact Abelian group the above integral coincides with the Haar integral on $A / H$. This proves the uniqueness of $\nu$. Since $S$ is an Abelian semigroup we know that there is at least one invariant mean on $m(S)$ and so also on $C(S)$. The proof of Theorem 1 is now complete.

Theorem 2. Let $S$ and $S^{\prime}$ be Abelian topological semigroups and let $f: S \rightarrow S^{\prime}$ be a continuous homomorphism of $S$ onto $S^{\prime}$. Let $F: C\left(S^{\prime}\right) \rightarrow C(S)$ be defined by

$$
\left(F x^{\prime}\right)(\sigma)=x^{\prime}(f(\sigma)), x^{\prime} \in C\left(S^{\prime}\right), \sigma \in S .
$$

Then $F^{*}$ carries the set of invariant means on $C(S)$ onto the set of invariant means on $C\left(S^{\prime}\right)$. Consequently the existence of many invariant means on $C\left(S^{\prime}\right)$ implies the existence of many invariant means on $C(S)$.

Proof. Let $\mu$ be an invariant mean on $C(S)$. Then $F^{*} \mu$ is a positive linear functional and since $\left(F^{*} \mu\right)\left(e^{\prime}\right)=\mu\left(F e^{\prime}\right)=\mu(e)=1$, we see that $F^{*} \mu$ is a mean on $C\left(S^{\prime}\right)$. Let $x^{\prime} \in C\left(S^{\prime}\right)$ and let $\sigma$ be an element of $S$. Then it can be easily seen that

$$
l_{\sigma}\left(F x^{\prime}\right)=F\left(l_{f \sigma}^{\prime} x^{\prime}\right) .
$$

If $\sigma^{\prime} \in S^{\prime}$ we may take $\sigma \in S$ such that $f \sigma=\sigma^{\prime}$. Then

$$
\begin{aligned}
\left(F^{*} \mu\right)\left(l_{\sigma^{\prime}}^{\prime} x^{\prime}\right) & =\mu\left[F\left(l_{f \sigma}^{\prime} x^{\prime}\right)\right]=\mu\left[l_{\sigma}\left(F x^{\prime}\right)\right] \\
& =\mu\left(F x^{\prime}\right)=\left(F^{*} \mu\right)\left(x^{\prime}\right) .
\end{aligned}
$$

Consequently $F^{*} \mu$ is an invariant mean on $C\left(S^{\prime}\right)$.

Suppose now that $\mu^{\prime}$ is an invariant mean on $C\left(S^{\prime}\right)$. Let

$$
C_{0}=\left\{F x^{\prime} \mid x^{\prime} \in C\left(S^{\prime}\right)\right\}
$$

and define $\mu_{0}$ on $C_{0}$ by

$$
\mu_{0}\left(F x^{\prime}\right)=\mu^{\prime}\left(x^{\prime}\right) .
$$


$\mu_{0}$ is well-defined since $F$ is a 1-1 mapping. Since $l_{\sigma}\left(F x^{\prime}\right)=F\left(l_{\rho \sigma}^{\prime} x^{\prime}\right)$ we conclude that $C_{0}$ is invariant under every $l_{\sigma}$. We next observe that $\mu_{0}$ is invariant on $C_{0}$ under all the operators $l_{\sigma}$. This follows from the following calculation:

$$
\begin{aligned}
\mu_{0}\left[l_{\sigma}\left(F x^{\prime}\right)\right] & =\mu_{0}\left[F\left(l_{f \sigma}^{\prime} x^{\prime}\right)\right]=\mu^{\prime}\left(l_{f \sigma}^{\prime} x^{\prime}\right) \\
& =\mu^{\prime}\left(x^{\prime}\right)=\mu_{0}\left(F x^{\prime}\right) .
\end{aligned}
$$

Thus we have a linear functional $\mu_{0}$ defined on an invariant subspace $C_{0}$ and invariant under all the operators $l_{\sigma}$. We now use a theorem of Silverman [3] to obtain an extension $\mu$ of $\mu_{0}$ which is an invariant mean on $C(S)$. It can be easily verified that $F^{*} \mu=\mu^{\prime}$.

It may be remarked that if $S$ and $S^{\prime}$ are completely regular spaces in which the translations are uniformly continuous mappings (this condition is satisfied if $S$ and $S^{\prime}$ are groups) and if $f: S \rightarrow S^{\prime}$ is uniformly continuous from $S$ onto $S^{\prime}$ then the above method can be used to prove that the existence of many invariant means on $U C\left(S^{\prime}\right)$, the space of bounded, real-valued uniformly continuous functions on $S$, implies the existence of many invariant means on $U C(S)$.

We say that an Abelian topological group $G$ has the property $P^{\prime}$ if there exists a countable subgroup $H$ and a symmetric neighborhood $V$ of 0 such that

(i) $(V+V) \cap H=\{0\}$,

(ii) $V$ is maximal among the symmetric neighborhoods of 0 which satisfy (i),

(iii) there exists a neighborhood $W$ of 0 such that $V+V+W$ meets $H$ at only finitely many points.

It may be observed that if $G$ contains a countable discrete subgroup $H$ we can easily find, by an application of Zorn's lemma, a symmetric neighborhood $V$ of 0 which satisfies (i) and (ii). Thus (iii) is the strong condition.

An Abelian topological group will be said to have property $P$ if $G$ or a factor group of $G$ has property $P^{\prime}$. The property $P$ is not very restrictive since many groups which can at all be expected to possess this property do possess it. Obviously an infinite discrete group $G$ has the property $P^{\prime}$ with $H$ any countable subgroup of $G, V$ constructed by Zorn's lemma and with $W=0$. Next any subgroup $G \neq\{0\}$ of the additive group of real numbers has the property $P^{\prime}$. Without loss of generality we assume that $1 \in G$ and we let $H$ be the cyclic subgroup generated by 1 . Then

$$
V=\{x \mid-1 / 2<x<1 / 2, x \in G\}=W
$$

satisfy (i), (ii) and (iii). Again any nonzero subgroup $G$ of the additive group of a normed linear space $X$ has property $P$. For let $0 \neq \alpha \in G$. We can find a linear functional $f$ such that $f(\alpha) \neq 0$. Thus $G$ would be mapped homomorphically onto a nonzero subgroup $f G$ of the additive group of real numbers 
which, as we have shown, possesses $P^{\prime}$. Thus $G$ has $P$. Finally any locally compact Abelian group $G$ which is not compact has the property $P$. To prove this we use the fact that $G$ is isomorphic to $R_{p} \times G_{1}$ [4] where $G_{1}$ is a group which contains a compact subgroup $H$ such that $G_{1} / H$ is discrete. If $p \neq 0$ then $R$, the additive group of real numbers, is a homomorphic image of $G$ and so $G$ has property $P$ since $R$ has property $P^{\prime}$. If $p=0, G_{1} / H$ must be infinite since otherwise $G$ would be compact. Since $G_{1} / H$ has $P^{\prime}$ it follows that $G$ has $P$.

Theorem 3. Let $G$ be an Abelian topological group having property $P$. Then there are many invariant means on $C(G)$.

Proof. By virtue of Theorem 2 we may assume that $G$ has $P^{\prime}$. Thus there exists a symmetric neighborhood $V$ of 0 and a countable subgroup $H$ such that

(i) $(V+V) \cap H=\{0\}$, (i),

(ii) $V$ is maximal among the symmetric neighborhoods of 0 which satisfy

(iii) there exists a neighborhood $W$ such that $(V+V+W) \cap H$ is a finite set.

We now take up the proof in several steps.

(a) It is obvious that if $v+h=v^{\prime}+h^{\prime}, v, v^{\prime} \in V ; h, h^{\prime} \in H$, then $v=v^{\prime}$ and $h=h^{\prime}$. This is a consequence of (i).

(b) If $g$ is not in

$$
\overline{V+H}
$$

then $0 \neq 2 g \in H$. To prove this we choose $W^{\prime}$, a symmetric neighborhood of 0 , such that $\left(g+W^{\prime}\right) \cap(H+V)=\varnothing$ and $W^{\prime}+W^{\prime} \subset V$. Then

$$
V^{\prime}=\left(g+W^{\prime}\right) \cup V \cup\left(-g+W^{\prime}\right)
$$

is a symmetric neighborhood of 0 which is larger than $V$. Consequently $V^{\prime}+V^{\prime}$ must meet $H$. It follows therefore that $2 g=w_{1}^{\prime}+w_{2}^{\prime}+h=v+h$. By (a) this $v$ is independent of $W^{\prime}$ and since $v \in W^{\prime}+W^{\prime}$ for every sufficiently small $W^{\prime}$, it follows that $v=0$ (the group topology is Hausdorff). Thus $2 g=h$. If $h=0$, then

$$
V^{*}=V \cup\left(g+W^{*}\right)
$$

will contradict the maximal character of $V$ where $W^{*}$ is a symmetric neighborhood of 0 such that

$$
\left(g+W^{*}\right) \subset(\overline{V+\bar{H}})^{c} \text { and } W^{*}+W^{*} \subset V .
$$

(c) Let $G_{2}$ be the set of elements $g \in G$ for which $2 g=0$. We claim that either 


$$
\overline{V+H}=G
$$

or $G_{2}$ is a neighborhood of 0 . To prove this take

$$
g \notin \overline{V+H}
$$

and choose an open neighborhood $U$ of 0 such that

$$
(g+U) \cap(\overline{H+V})=\varnothing, \quad U+U \subset V .
$$

Take any $u \in U$. Then

$$
g+u \notin \overline{V+H}
$$

and therefore $2 g+2 u=h$. Since $2 g=h^{\prime}$, it follows from (a) that $2 u=0$. Thus $G_{2}$ contains $U$ and our assertion is proved.

(d) We will now construct two subsets $A$ and $B$ of $H$ which have the following properties:

(i) given any finite subset $\left\{h_{1}, \cdots, h_{n}\right\}$ of elements of $H$ there exists an $h \in H$ such that $h_{1}+h, \cdots, h_{n}+h$ are all in $A$ and an $h^{\prime}$ such that $h_{1}+h^{\prime}$, $\cdots, h_{n}+h^{\prime}$ are in $B$.

(ii) $(A+V+W) \cap(B+V)=\varnothing$.

$(V+V+W) \cap H$ is finite. Let its members be $h^{(1)}, \cdots, h^{(l)}$. Let us first assume that $H$ is finitely generated. Consequently, by the fundamental theorem on finitely generated Abelian groups, there exist $h_{1}, \cdots, h_{t}$ in $H$ and a finite subgroup $\Phi$ of $H$ such that every element $h$ of $H$ can be uniquely written in the form

$$
\lambda_{1} h_{1}+\cdots+\lambda_{t} h_{t}+\phi ; \quad \lambda_{i} \text { integers, } \phi \in \Phi .
$$

We shall call $\lambda_{1}, \cdots, \lambda_{t}$ the co-ordinates of $h$. We define $A_{1}$ to be $\Phi$. Suppose that finite subsets $A_{1}, A_{2}, \cdots, A_{p}$ of $H$ have been defined. Let $\mu_{p}$ be an integer larger than the absolute value of every co-ordinate of every member of the finite set

$$
\left\{h^{(i)}+h \mid 1 \leqq i \leqq l, h \in \bigcup_{j=1}^{p} A_{j}\right\}
$$

and define $A_{p+1}$ by

$$
A_{p+1}=\left\{h=\sum_{i=1}^{t} \lambda_{i} h_{i}+\phi\left|\phi \in \Phi, \mu_{p} \leqq\right| \lambda_{1}|, \cdots,| \lambda_{t} \mid \leqq \mu_{p}+10^{p}\right\} .
$$

We let

$$
A=\bigcup_{j=1}^{\infty} A_{2 j-1} \quad \text { and } \quad B=\bigcup_{j=1}^{\infty} A_{2 j}
$$


It can be easily verified that conditions (i) and (ii) are satisfied.

Suppose now that $H$ is not finitely generated. Since $H$ is countable, we enumerate the elements of $H$ as $h^{(1)}, \cdots, h^{(r)}, \cdots$ where $h^{(1)}, \cdots, h^{(l)}$ are the members of $H$ which are in $V+V+W$. We define sets $A_{1}, A_{2}, \cdots$, $A_{p}, \cdots$, not necessarily finite, in the following manner.

Let $A_{1}$ be the subgroup generated by $h^{(1)}, \cdots, h^{(l)}$. Suppose $A_{1}, A_{2}, \cdots$, $A_{p}$ have been defined in such a way that $\mathrm{U}_{j=1}^{p} A_{j}$ is a finitely generated subgroup $H_{p}$ of $H$. $H_{p}$ being not equal to $H$, let $h$ be the first among $h^{(1)}, \cdots$, $h^{(r)}, \ldots$ which is not in $H_{p}$ and let $A_{p+1}$ consist of those elements of the subgroup generated by $H_{p}$ and $h$ which are not in $H_{p}$.

We now define

$$
A=\bigcup_{j=1}^{\infty} A_{2 j-1}, \quad B=\bigcup_{j=1}^{\infty} A_{2 j} .
$$

It can again be easily verified that $A$ and $B$ have the properties (i) and (ii).

(e) We now prove a result which we will need in the next step.

Let $F_{0}$ and $F_{1}$ be subsets of an Abelian topological group $G$ such that there exists a symmetric neighborhood $W$ of 0 such that $\left(F_{0}+W\right) \cap F_{1}=\varnothing$. Then there exists $x$ in $C(G)$ such that $x(g)=-1$ if $g \in F_{0}$ and $x(g)=1$ if $g \in F_{1}$.

To prove this we first note that we can assume $F_{0}$ and $F_{1}$ to be closed, for otherwise we replace $F_{0}$ by $\bar{F}_{0}, F_{1}$ by $\bar{F}_{1}$ and $W$ by $W_{1 / 3}$ where $W_{1 / 3}$ stands for any symmetric neighborhood of 0 such that $W_{1 / 3}+W_{1 / 3}+W_{1 / 3} \subset W$.

Let now $V_{1}=F_{1}^{c}$. We define $V_{1 / 2}$ to be $F_{0}+W_{1 / 3}$. Then $F_{0} \subset V_{1 / 2} \subset \bar{V}_{1 / 2}$ $\subset V_{1}$. Since $\left(F_{0}+W_{1 / 3}\right) \cap V_{1 / 2}^{c}=\varnothing=\left(\bar{V}_{1 / 2}+W_{1 / 3}\right) \cap F_{1}$ we can repeat the above process with the pairs of closed sets $\left(F_{0}, V_{1 / 2}^{c}\right)$ and $\left(\bar{V}_{1 / 2}, F_{1}\right)$ to get sets $V_{1 / 4}$ and $V_{3 / 4}$. We continue this process to get an open set $V_{t}$ for each $t$ of the form $\left(m / 2^{n}\right), 0<t \leqq 1$, such that

$$
F_{0} \subset V_{t}, \quad \bar{V}_{t} \subset V_{1} \text { and } \bar{V}_{t} \subset V_{t^{\prime}} \text { if } t<t^{\prime} .
$$

We define $y \in C(G)$ as follows:

$$
y(g)=\left\{\begin{array}{c}
1 \text { if } g \notin \underset{t}{U} V_{t}, \\
\operatorname{Inf}_{g \in V_{t}} t \text { otherwise. }
\end{array}\right.
$$

Thus $y(g)=0$ if $g \in F_{0}$ and $y(g)=1$ if $g \in F_{1}$. Then $x \in C(G)$ defined by

$$
x(g)=2 y(g)-1
$$

satisfies our requirements.

(f) Suppose that

$$
\overline{H+V}=G .
$$

By means of (ii) of (d) and the result proved in (e) we construct $x_{0} \in C(G)$ 
such that $x_{0}$ takes the value -1 on $A+V$ and the value 1 on $B+V$. For $x \in C(G)$, let

$$
p(x)=\operatorname{Inf}_{\sigma_{1}, \ldots, \sigma_{n}} \operatorname{Sup} \frac{1}{n} \sum_{j=1}^{n} x\left(\sigma_{j}+\sigma\right)
$$

where the Inf is taken over all finite sequences of elements of $G$. If

$$
\sigma_{1}, \cdots, \sigma_{n} \in G=\overline{H+V}
$$

we can find $g$ such that $\sigma_{1}+g, \cdots, \sigma_{n}+g$ are all in $H+V$. So we may assume that $\sigma_{1}, \cdots, \sigma_{n}$ are in $H+V$. Let $\sigma_{i}=h_{i}+v_{i}, 1 \leqq i \leqq n$. It follows from (i) of (d) that $p\left(x_{0}\right)$ and $p\left(-x_{0}\right)$ are both larger than or equal to 1. Thus $p\left(x_{0}\right)$ $\neq-p\left(-x_{0}\right)$ and so by Lemma 1 on page 37 of [2] we see that there are many invariant means on $C(G)$.

$\left(f^{\prime}\right)$ Suppose that

$$
\overline{H+V} \neq G \text {. }
$$

In this case $G_{2}$ is an open and closed subgroup of $G$ and so $G / G_{2}$ is discrete. If $G / G_{2}$ is infinite, there are many invariant means on $G / G_{2}$ [1] and hence also on $G$. So assume that $G / G_{2}$ is finite. Let $V_{2}=V \cap G_{2}, W_{2}=W \cap G_{2}$ and $H_{2}=H \cap G_{2}$.

If possible let

$$
\overline{H_{2}+V_{2}} \neq G_{2} \text {. }
$$

Then there exists a nonempty set $U_{2}$, open in $G_{2}$ and so also in $G$, such that $U_{2} \cap\left(H_{2}+V_{2}\right)=\varnothing$. We claim that $U_{2} \cap(H+V)=\varnothing$ for otherwise there exists $u_{2} \in U_{2}, h \in H$ and $v \in V$ such that $u_{2}=h+v$. But then $0=2 u_{2}=2 h+2 v$. Consequently $2 h=2 v=0$, i.e., $h \in H_{2}$ and $v \in V_{2}$. This however implies that $u_{2} \in H_{2}+V_{2}$ which is not true. Thus our claim is established. Since $U_{2}$ is open it follows that

$$
U_{2} \cap(\overline{H+V})=\varnothing .
$$

This however contradicts (b). Therefore

$$
\overline{H_{2}+V_{2}}
$$

must be equal to $G_{2}$.

Since $G / G_{2}$ is finite, $H_{2}$ must be infinite. Moreover $\left(V_{2}+V_{2}+W_{2}\right) \cap H_{2}$ is finite. Therefore we can construct a function $x_{2} \in C\left(G_{2}\right)$ for which $p_{2}\left(x_{2}\right)$ and $p_{2}\left(-x_{2}\right)$ are $\geqq 1$ where $p_{2}$ has the obvious meaning. We now define $x \in C(G)$ by 
1962] THE INVARIANT MEAN ON ABELIAN TOPOLOGICAL SEMIGROUPS 411

$$
x\left(\sigma+g_{2}\right)=x_{2}\left(g_{2}\right)
$$

where $\sigma$ belongs to a fixed set of representatives $\bmod G_{2}$. We can easily satisfy ourselves that for this $x, p(x) \neq-p(-x)$.

This completes the proof of the theorem.

\section{BIBLIOGRAPHY}

1. M. M. Day, Amenable semigroups, Illinois J. Math. 1 (1957), 509-544.

2. Indar S. Luthar, Uniqueness of the invariant mean on an Abelian semi-group, Illinois J. Math. 3 (1959), 28-44.

3. R. J. Silverman, Invariant linear functions, Trans. Amer. Math. Soc. 81 (1956), 411-424.

4. André Weil, L'integration dans les groupes topologiques et ses applications, Hermann, Paris, 1950.

Panjab University,

Chandigarh, India 